\title{
Statins: the high risks of discontinuation and large benefits of continuation
}

Charles H. Hennekens ${ }^{1-3}$, Joanna Drowos ${ }^{1,2,4}$

\author{
${ }^{1}$ Charles E. Schmidt College of Medicine, Florida Atlantic University, USA \\ 2Department of Preventive Medicine, Nova Southeastern University College \\ of Osteopathic Medicine, USA \\ ${ }^{3}$ Department of Family Medicine and Community Health, University of Miami Miller \\ School of Medicine, USA \\ ${ }^{4}$ Palm Beach County Health Department, USA
}

Submitted: 12 December 2011

Accepted: 15 December 2011

Arch Med Sci 2011; 7, 6: 931-932

DOI: 10.5114/AOMS.2011.26602

Copyright (c) 2011 Termedia \& Banach

In their mini-review/short communication in this issue of the Archives of Medical Science, Dr. Paraskevas et al. [1] draw proper attention to the consequences of statin discontinuation. They focus primarily on the cerebrovascular disease but their conclusion that absolute benefits of discontinuation are small and the absolute risks are high is consistent with a large and robust totality of evidence.

The Cholesterol Treatment Trialist's Collaboration [2, 3] published two worldwide and comprehensive meta-analyses of randomized data. The first, among about 90,000 participants in 14 randomized trials, showed that statin therapy produced statistically significant and clinically important reductions in myocardial infarction, stroke, cardiovascular death and total mortality and a remarkable safety profile. The second, among 170,000 participants in 26 randomized trials, showed that further reductions by statins in low density lipoprotein (LDL) cholesterol safely produce definite further reductions in the incidence of myocardial infarction, stroke and revascularization. Specifically, each $1.0 \mathrm{mmol} / \mathrm{I}$ reduction in LDL cholesterol produces a statistically significant and clinically important reduction in the annual rate of these major vascular events by just over $20 \%$. There was no evidence of any threshold within the cholesterol range studied, suggesting that reduction of LDL cholesterol by $2-3 \mathrm{mmol} / \mathrm{l}$ would reduce risk by about $40-50 \%$. Thus, the recent randomized trials of statins using higher doses against lower doses as the active comparator arm have contributed importantly relevant information to two long standing hypotheses [4]. The first is that lower is better and the prior totality of evidence included basic research and observational epidemiological studies, including in rural China where the average total cholesterol is less than $3.7 \mathrm{mmol} / \mathrm{l}$ and those with total cholesterol levels that are 10\% lower had significantly decreased risks of occlusive vascular events [5]. The second is that there is no threshold and the meta analysis of the data from randomized trials with active comparators in secondary prevention contribute importantly relevant data to this hypothesis. In terms of primary prevention the JUPITER trial also contributes the most importantly relevant data as the randomized population had no prior events and a 10 year risk of a first CHD event of $16-18 \%$ [6]. Further, at baseline the LDL was about 3.0 and subjects assigned to $20 \mathrm{mg}$ rosuvastatin achieved a greater than
Corresponding author: Charles H. Hennekens $\mathrm{MD}, \mathrm{PhD}$

Charles E. Schmidt College of Medicine Florida Atlantic University 2800 S. Ocean Blvd. PHA Boca Raton, FL 33432 USA Phone: 5613938845 Fax: 5616202182

E-mail: chenneke@fau.edu 
$50 \%$ reduction in LDL to about 1.4 . The trial was terminated early after a median treatment and follow up of about 1.9 years due to a $44 \%$ statistically extreme reduction in the primary composite endpoint. In JUPITER there were also statistically significant reductions in $\mathrm{MI}$, stroke, and revascularizations and this was the first primary prevention trial to demonstrate a statistically significant and clinically important reduction in total all cause mortality. It seems most plausible that the large reductions in LDL to levels far below those that had ever been studied in primary prevention were critical components of the favorable outcomes.

In conclusion, in this issue of the Archives of Medical Science, Dr. Paraskevas et al. contribute an added dimension to the risks of discontinuation of statins. The risks are high of statin discontinuation so any decision to do so should be made by the individual health care provider and each of his or her patients. The general goal should be to avoid discontinuation of statins whenever possible.

\section{Acknowledgments}

Disclosures: Professor Hennekens was funded by the Charles E. Schmidt College of Medicine at Florida Atlantic University as principal investigator on 2 investigator-initiated research grants funded to Florida Atlantic University by Bayer; serves in an advisory role to investigators and sponsors as Chair of independent Data and Safety Monitoring Boards for Actelion, Amgen, Bristol- Myers Squibb, and Sunovion, and as a member of independent Data and Safety Monitoring Boards for Bayer and the Canadian Institutes of Health Research; serves in an advisory role to the US Food and Drug Administration, US National Institutes of Health, and UpToDate; serves as an independent scientist in an advisory role to legal counsel for GlaxoSmithKline and Stryker; serves as a speaker for the Association of Research in Vision and Ophthalmology, National Association for Continuing Education, PriMed, and the International Atherosclerosis Society; receives royalties for authorship or editorship of 3 textbooks and as co-inventor on patents for inflammatory markers and cardiovascular disease that are held by Brigham and Women's Hospital; and has an investment management relationship with the West-Bacon Group within SunTrust Investment Services, which has discretionary investment authority and excludes investments in pharmaceutical or device companies.

Dr. Drowos has no disclosures.

\section{References}

1. Paraskevas KI, Koupidid S, Gentimi F, Tzovaras AA. Statin discontinuation: counterbalancing the benefits with the potential risks. Arch Med Sci 2011; 7: 1076-7.

2. Cholesterol Treatment Trialists' (CTT) Collaborators. Efficacy and Safety of cholesterol-lowering treatment: prospective meta-analysis of data from 90,056 participants in 14 randomized trials of statins. Lancet 2005; 366 1267-78.

3. Cholesterol Treatment Trialists' (CTT) Collaborators. Efficacy and safety of more intensive lowering of LDL cholesterol: a meta-analysis of data from 170,000 participants in 26 randomised trials. Lancet 2010; 376: 1670-81

4. Hennekens $\mathrm{CH}$, Schneider WR. The need for wider and appropriate utilization of aspirin and statins in the treatment and prevention of cardiovascular disease. Expert Rev Cardiovasc Ther 2008; 6: 95-107.

5. Chen Z, Jiang L, ChenY et al. Addition of clopidogrel to aspirin in 45,852 patients with acute myocardial infarction: randomised placebo-controlled trial. Lancet 2005; 366: 1607-21.

6. Ridker PM, Danielson E, Fonseca FAH, et al. for the JUPITER Study Group. Rosuvastatin to prevent vascular events in men and women with elevated C-reactive protein. N Engl J Med 2008; 359: 2195-207. 